\title{
IMPROVING THE RATE OF THE TRIPLE ALPHA PROCESS
}

\author{
C. Tur*, S. M. Austin \\ National Superconducting Cyclotron Laboratory \\ Michigan State University, 1 Cyclotron Laboratory, East Lansing, MI 48824-1321 \\ Joint Institute for Nuclear Astrophysics \\ E-mail: tur@nscl.msu.edu
}

\author{
A. Wuosmaa, J. Lighthall, S. Marley, N. Goodman, and J. J. Bos \\ Western Michigan University \\ Physics Department, 1903 W. Michigan Avenue, Kalamazoo, MI 49008-5252
}

\begin{abstract}
The rate of the triple alpha process, which plays a central role in the production of ${ }^{12} \mathrm{C}$ in stars, is known with an accuracy of about $12 \%$. Variations within the $\pm 12 \%$ errors in this rate can cause significant changes in the determination of the mass of the iron core in core-collapse supernovae and the composition of the material later ejected in the interstellar medium, as well as a factor of two change in the surface abundance of ${ }^{12} \mathrm{C}$ in light $\mathrm{ABG}$ stars.

The triple alpha experiment presented here is a collaborative effort between the Western Michigan University (WMU) and the National Superconducting Cyclotron Laboratory (NSCL) and aims at reducing the uncertainty on the knowledge of this rate to about $6 \%$ by measuring more accurately than has been done in the past the pair branch for the $7.654 \mathrm{MeV}$ state in ${ }^{12} C$. This state is excited by inelastic proton scattering $\left({ }^{12} C\left(p, p^{\prime}\right){ }^{12} C(7.654 \mathrm{MeV})\right)$, taking advantage of a strong resonance at a bombarding energy of about $10.6 \mathrm{MeV}$ and a scattering angle of 135 degrees in the laboratory. The decay pairs are detected in an almost $4 \pi$ scintillator system surrounding the target. The protons are produced using the Tandem accelerator at Western Michigan University and detected by silicon detectors at 135 degrees in the lab. The pair branch is given by the ratio of the number of electron-positron pairs detected in the plastic scintillators in coincidence with the protons in the $7.654 \mathrm{MeV}$ silicon spectra to the number of those protons. A reduction in the gamma ray background, mainly due to the cascade gamma decay of the $7.654 \mathrm{MeV}$ state through the $4.44 \mathrm{MeV}^{+}$state, is achieved by a coincidence requirement between a thin scintillator tube and the large block of plastic scintillator surrounding it. The measurement is expected to take place in the summer of 2006.
\end{abstract}

International Symposium on Nuclear Astrophysics - Nuclei in the Cosmos - IX

June 25-30 2006

CERN, Geneva, Switzerland

* Speaker. 


\section{The triple alpha process and the experimental determination of its rate}

The triple alpha process, which produces ${ }^{12} C$, is one of two fundamental reactions essentially defining the helium burning phase in stars. The ${ }^{12} C(\alpha, \gamma){ }^{16} O$ reaction is the other one; it competes with the triple alpha process by converting carbon into oxygen, thereby determining the relative abundance of carbon to oxygen at the completion of helium burning. Since those two reactions are the main source of carbon and oxygen, the ratio of their rates also determines the relative abundance of carbon to oxygen in the universe.

The rate $r_{3 \alpha}$ for the triple alpha reaction can be written as

$$
r_{3 \alpha} \propto \Gamma_{\text {rad }} \exp \left(-Q_{3 \alpha} / k T\right),
$$

where $\mathrm{T}$ is the temperature, $Q_{3 \alpha}$ the energy released in the decay ${ }^{12} C(7.654) \rightarrow 3 \alpha$, and $\Gamma_{\text {rad }}=$ $\Gamma_{\pi}+\Gamma_{\gamma}$ is the radiative width of the Hoyle state $\left(\Gamma_{\pi}\right.$ denotes the partial width for the E0 internal pair transition to the ground state and $\Gamma_{\gamma}$, the partial width for the cascade gamma transition to the ground state). Experimentally, $\Gamma_{\text {rad }}$ is determined as the product of three independently measured quantities:

$$
\Gamma_{\text {rad }}=\left(\frac{\Gamma_{\text {rad }}}{\Gamma}\right)\left(\frac{\Gamma}{\Gamma_{\pi}}\right)\left(\Gamma_{\pi}\right)
$$

where $\Gamma$ is the total width of the $7.654 \mathrm{MeV}$ state. Thus, there are four experimental parameters to be determined in order to know $r_{3 \alpha}$.

The value of $Q_{3 \alpha}$ is well known from 6 measurements to be $379.38 \pm 0.20 \mathrm{keV}$ ([13], [14], [15], [16], [17], [18]), contributing only $1.2 \%$ to the fractional error on $r_{3 \alpha}$ for temperatures of $2 \times 10^{8} \mathrm{~K}$. $\frac{\Gamma_{r a d}}{\Gamma}$ has a value of $(4.12 \pm 0.11) \times 10^{-4}$ (error of $\left.2.7 \%\right)$ known from 8 measurements of which one has been excluded here ([5], [5] (excluded), [7], [8], [9], [10], [11], [12]). The value of the pair branch, $\frac{\Gamma_{\pi}}{\Gamma}$, is known to be $(6.74 \pm 0.62) \times 10^{-6}$ (error of 9.2\%) from 3 measurements ([19], [20], [21]). The pair branch is thus a very small quantity, which makes its experimental determination challenging. The most widely known value for the fourth parameter, $\Gamma_{\pi}$, is $(60.5 \pm 3.9) \times 10^{-6} \mathrm{eV}$ (error of 6.4\%) known from 2 measurements ([22], [23]). The errors on those 4 parameters combine to give an error on $r_{3 \alpha}$ of $11.6 \%$. A new value and error for $\Gamma_{\pi}$ have recently been claimed by $\mathrm{H}$. Crannell et al. (24]) based on the analysis of all extant electron scattering data. The new value is $(52.0 \pm 1.4) \times 10^{-6} \mathrm{eV}$ with an error of $2.7 \%$. This new error on $\Gamma_{\pi}$ together with the known errors on the 3 remaining parameters combine to give an error of $10 \%$ on $r_{3 \alpha}$. All in all, the biggest uncertainty $(9.2 \%)$ is due to $\frac{\Gamma_{\pi}}{\Gamma}$, which the present experiment is aimed at reducing.

\section{The experiment}

The measurement is an improvement of an experiment by Robertson et al. ([20]). The detector (Figure 2) comprises an outer BC404 scintillator block with a central cylindrical hole. The block is divided into four quadrants optically isolated from each other. Each quadrant is viewed by a light guide and photo-multiplier tube. Within the central hole fits a cylindrical BC404 scintillator tube, within which there is a smaller cylindrical tube of non-scintillating plastic (acrylic), there to prevent scattered protons from entering the scintillators. The inner scintillator tube is optically isolated from the outer block and is viewed by a fifth photo-multiplier tube (not represented on 


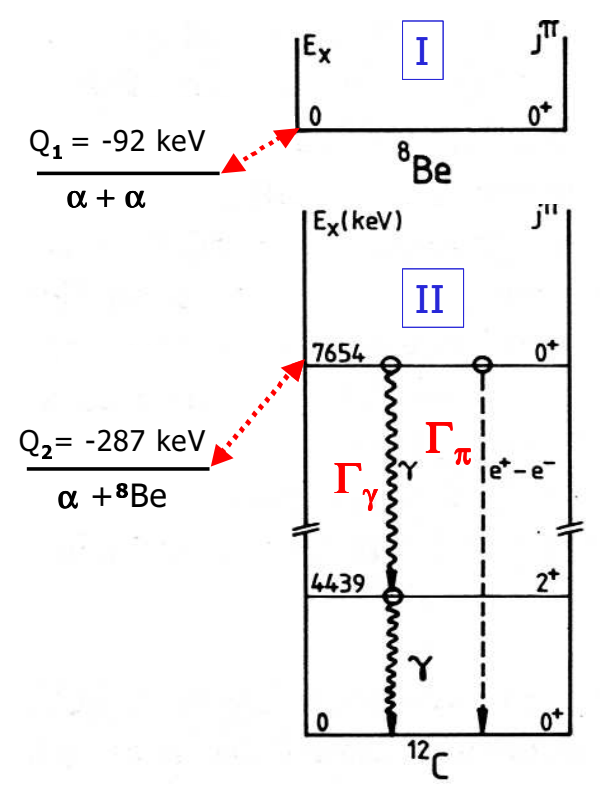

Figure 1: The triple alpha process and the decay of the Hoyle state via internal conversion or cascade gamma pair emission.

the figure). A $10.6 \mathrm{MeV}$ proton beam from the Tandem accelerator at WMU impinges on a ${ }^{12} \mathrm{C}$ target at the center of the detector, exciting both the $4.44 \mathrm{MeV}$ and the $7.654 \mathrm{MeV}$ states of ${ }^{12} C$. Scattered protons are detected in two silicon detectors placed outside the scintillator block at 135 degrees to the beam. The pair branch is then given by the ratio of the number of electron-positron pairs detected in the plastic scintillators in coincidence with the protons in the $7.654 \mathrm{MeV}$ peak of the silicon spectra to the number of such scattered protons. A coincidence requirement between the inner scintillator tube and the outer block greatly reduces the gamma ray background, mainly due to the cascade gamma decay of the $7.654 \mathrm{MeV}$ state through the $4.44 \mathrm{MeV}$ state. Observation of the pair decay of the $0^{+}$level at $6.05 \mathrm{MeV}$ in ${ }^{16} \mathrm{O}$ will provide a precise calibration of the detector and line shapes of the experiment. This level has a $100 \%$ decay branch for internal pair transition to the ground state. The $e^{+} e^{-}$pairs from the $6.05 \mathrm{MeV}$ state will be observed by bombarding a target containing ${ }^{16} \mathrm{O}$ (Formvar for instance) with $10.6 \mathrm{MeV}$ protons. The value of their observed branching ratio should provide the efficiency for detecting electron-positron pairs. A monte carlo simulation of this decay was done. Once the peak position is determined from the simulations, a fit to the data should give the energy calibration for the pair detector.

A major limitation in the Robertson et al. measurement was statistics. The present experiment will not be subjected to any strict run-time constraints, which should greatly reduce the statistical limitation. In addition, fewer pile-up and chance coincidences are expected owing to the dc beam instead of a pulsed one, faster scintillators and electronics than 30 years ago, greater granularity of the detector, and lower instantaneous beam current. The inner scintillator tube being thinner than the one used for the old experiment, less gamma background should ensue. An increased target purity (99.9999\%) should also result in reduced background due to target contaminants. For all these reasons, a 5\% measurement of the pair branch should be achievable (down from the current uncertainty of $9.2 \%$ ), which would decrease the corresponding error on the triple alpha rate to 


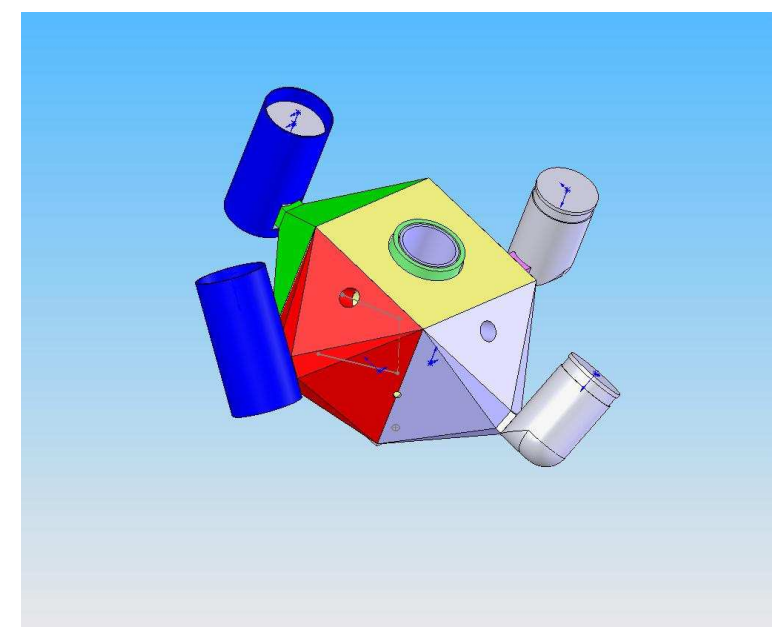

Figure 2: The triple alpha detector. The central scintillator block is $10 \mathrm{~cm} \times 10 \mathrm{~cm}$, the inner diameter of the $3 \mathrm{~mm}$ thick scintillator tube is $2 \mathrm{~cm}$. The beam entrance hole (on the corner of the scintillator block) and the exit holes for the scattered protons are also shown.

about $6 \%$.

\section{Why is a better accuracy on $r_{3 \alpha}$ needed?}

A more accurate determination of the triple alpha rate is needed to predict more precisely the amount of ${ }^{12} \mathrm{C}$ dredged-up to the surface of light Asymptotic Giant Branch (ABG) stars and its uncertainty affects the size of the iron core of a core-collapse supernova progenitor at the onset of core-collapse, as well as the intermediate mass nuclei produced in supernovae explosions.

\subsection{AGB stars}

Towards the end of their lives, after the hydrogen and helium burning phases, intermediate mass stars (with a mass range from about that of the sun to about 8 to 10 solar masses), go through the Asymptotic Giant Branch (AGB) phase ([2]). During this phase, the star has a carbon-oxygen degenerate core and burns helium and hydrogen in shells surrounding the core. The helium and hydrogen burning shells are separated by a non-burning intershell region $\left(75 \%{ }^{4} \mathrm{He}\right.$ and $\left.22 \%{ }^{12} \mathrm{C}\right)$. A convective envelope surrounds the hydrogen burning shell and is characterized by very low densities, the consequence of which is that material from the envelope can easily be blown into the interstellar medium by stellar winds. Every few 10,000 years, thermal flashes, induced by the helium burning shell, dredge up ${ }^{12} C$ (among other elements) into the intershell region and the convective envelope from which it can be blown off the star and into the interstellar medium.

A study conducted by Herwig and Austin ([1] [ [3]) on low mass ABG stars shows that stronger helium flashes are induced resulting in a more efficient, deeper dredge up, which itself leads to an increased enrichment of the envelope in ${ }^{12} \mathrm{C}$ by a factor as large as two, when either the triple alpha rate (as given by NACRE) is increased by its uncertainty or when the ${ }^{14} N(p, \gamma){ }^{15} O$ rate is decreased by its uncertainty. The ${ }^{14} N(p, \gamma){ }^{15} O$ rate has recently been measured to $5 \%$ ([四). Clearly, this implies the need for a better accuracy for the triple alpha rate in order to resolve the issue, at least as far as nuclear reaction rates are concerned. 


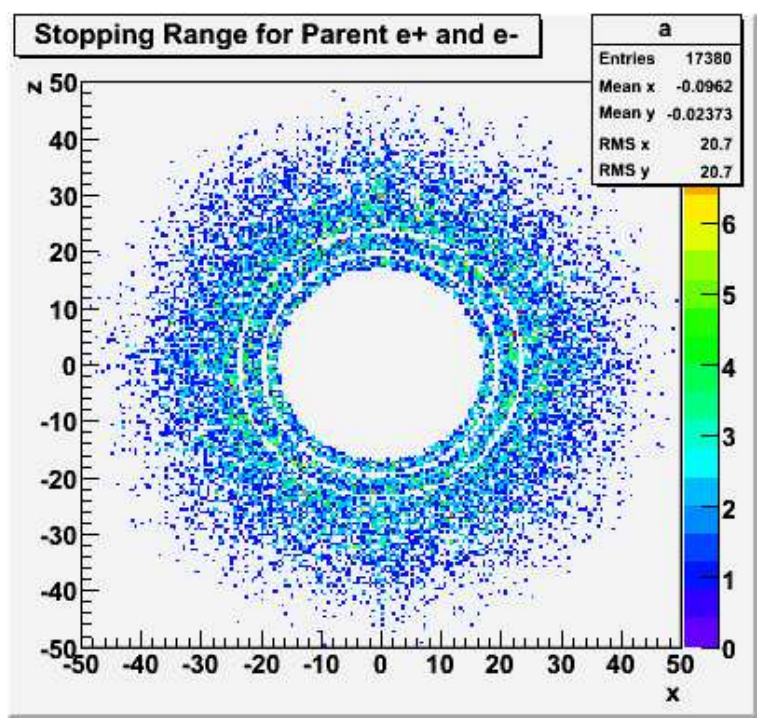

Figure 3: The $\mathrm{x}$ and $\mathrm{z}$ coordinates of the stopping point of the $e^{+}$and $e^{-}$in the detector. The ranges of the $\mathrm{x}$ and $\mathrm{z}$ axes are the size of the detector (in $\mathrm{mm}$ ).

\subsection{Core-collapse supernova progenitors}

Studies by Woosley et al. ([25]), suggest a sensitive dependence of the size of the iron core of a massive star (mass greater than 10 solar masses) at the onset of core-collapse on the ratio $R=r_{3 \alpha} / r_{12} C(\alpha, \gamma){ }^{16} O$. Varying the ${ }^{12} C(\alpha, \gamma){ }^{16} O$ rate by $\pm 10 \%$ around the central value of 1.2 times the rate suggested by Buchmann in 1996 (this value is chosen based on nucleosynthesis arguments of the medium-weight elements by such massive stars) generates an uncertainty of 0.2 solar masses for a 25 solar mass star. This uncertainty seems important, since an energy similar to the energy released in a supernova explosion is required to dissociate that much matter into nucleons. The current uncertainty on $\mathrm{R}$ is much worse than $10 \%$ : the triple alpha rate is known to 10 to $12 \%$, while the ${ }^{12} C(\alpha, \gamma){ }^{16} O$ rate is at best known to $20 \%$. Clearly a better knowledge of both reaction rates is necessary. Bringing the error on the triple alpha rate down to $6 \%$ will be a step forward in that direction.

\section{The simulations}

Extensive simulations were done using GEANT4 in order to have confidence in the design of the detector and to be used as a guidance for the measurement itself. An outer block made of BC404 scintillator with a cylindrical hole at its center and divided into 4 quadrants was implemented in the detector geometry. A thin BC404 scintillator tube and a thin acrylic tube were inserted in the central cylindrical hole according to the design of the detector. A generator was placed in the middle of the detector, capable of throwing cascade gamma pairs (gammas of $3.21 \mathrm{MeV}$ and $4.44 \mathrm{MeV}$ in energy, randomly oriented in $4 \pi$ ) or $e^{+} e^{-}$pairs having the correct energy-angular correlation for an E0 transition from the Hoyle state to the ground state of ${ }^{12} C$.

Plots were generated for the stopping range of the $e^{+}$and $e^{-}$in the detector (Figure 3), as well as for energy depositions (Figure 4). The study showed that essentially all the $e^{+} e^{-}$pairs from the 

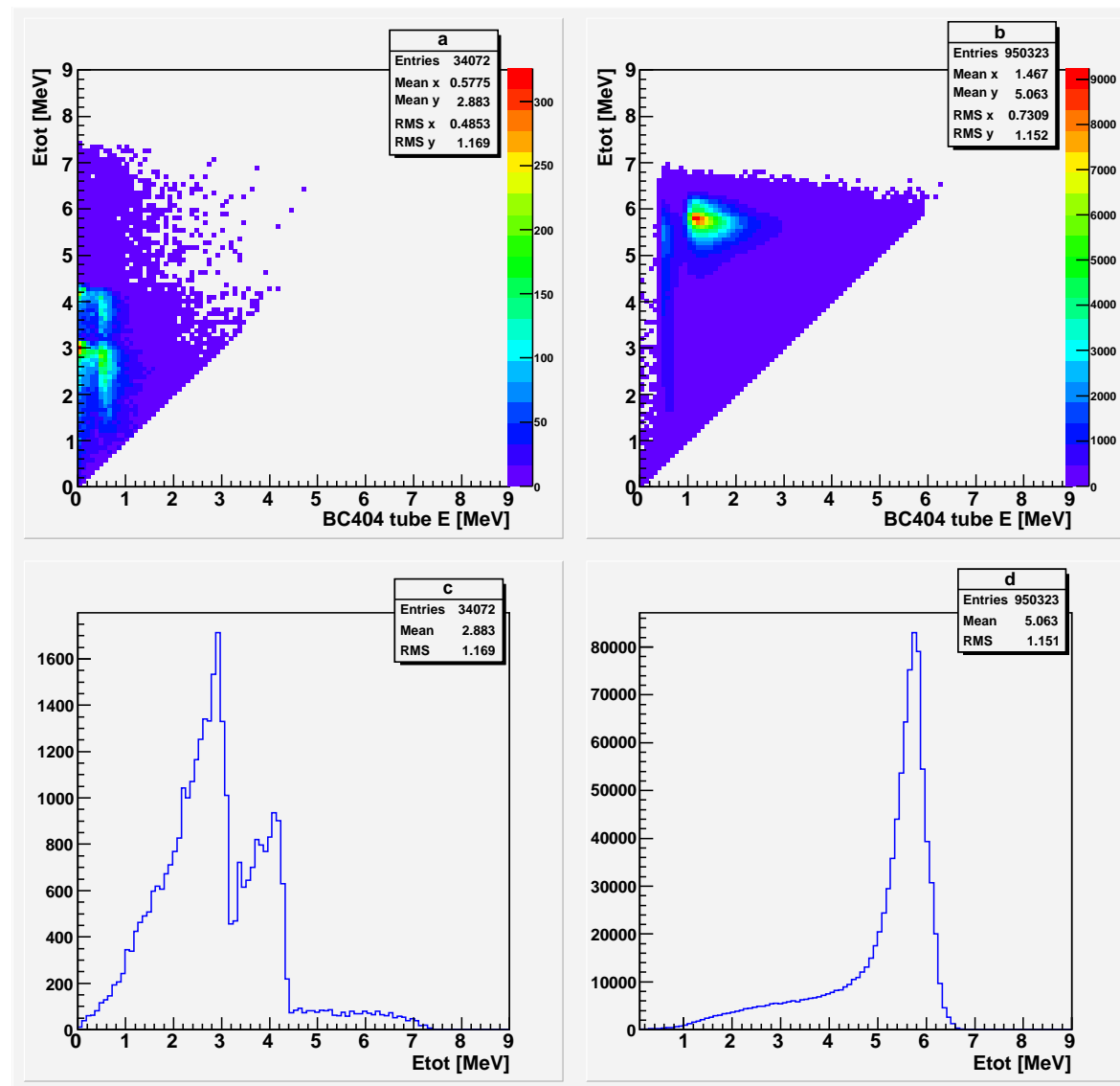

Figure 4: Energy depositions of the thrown cascade gamma and the $e^{+} e^{-}$pairs from the Hoyle state in the scintillators. The scatter plots represent the total energy (energy deposited in the scintillator tube and the outer block) versus the energy deposited in the scintillator tube only (left: cascade gamma case, right: $e^{+} e^{-}$ pairs case). The two lower plots show the counts versus the energy deposition in the scintillator tube (same cases as above on the left and right hand sides). A coincidence is required between the scintillator tube and the outer block. One million pairs are thrown in both cases.

decay of the Hoyle state, which do not escape through the central hole, would be stopped in the detector and that most of them would stop near the center and away from the corners of the outer block where light collection is not optimum. The energy deposition plots confirmed the fact the total energy deposited by most of the $e^{+} e^{-}$pairs was well separated from the total energy deposited by most of the cascade gamma pairs. Thus, a combination of cuts on the energy deposited in the various parts of the detector allows one to eliminate a very large fraction of the cascade gamma pairs which pass the basic coincidence requirement between the scintillator tube and the outer block.

\section{Conclusion}

The detector has been built at the NSCL and has already undergone extensive tests. The measurement is expected to be carried out during the summer of 2006 using the Tandem accelerator 
at WMU. It should yield a 5\% error on the internal pair conversion branch of the Hoyle state, leading to an accuracy of about $6 \%$ on the rate of the fundamental triple alpha process.

\section{Acknowledgments}

This work was supported in part by the US National Science Foundation grants PHY01-10253 and PHY02-16783, the latter funding the Joint Institute for Nuclear Astrophysics (JINA), a National Science Foundation Physics Frontier Center and the US Department Of Energy grant DEFG02-04R41320.

\section{References}

[1] F. Herwig and S. M. Austin, Astrophys. J. 613 (2004) L73.

[2] F. Herwig, Аnпи. Rev. Astron. Astrophys. 43 (2005) 435.

[3] F. Herwig, S. M. Austin, and J. C. Lattanzio, Phys. Rev. C 73 (2006) 025802.

[4] A. Lemut et al., [arXiv:nucl-ex/0602012].

[5] D. E. Alburger, Phys. Rev. 124 (1961) 193.

[6] P. A. Seeger and R. W. Kavanagh, Nucl. Phys. 46 (1963) 577.

[7] I. Hall and N. W. Tanner, Nucl. Phys. 53 (1964) 673.

[8] D. Chamberlin, D. Bodansky, W. W. Jacobs, and D. L. Oberg, Phys. Rev. C 9 (1974) 69.

[9] C. N. Davids, R. C. Pardo, and A. W. Obst, Phys. Rev. C 11 (1975) 2063.

[10] H. -B. Mak, H. C. Evans, G. T. Ewan, A. B. McDonald, and T. K. Alexander, Phys. Rev. 12 (1975) 1158 .

[11] A. W. Obst and W. J. Braithwaite, Phys. Rev C 13 (1976) 2033.

[12] R. G. Markham, S. M. Austin, and M. A. M. Shahabuddin, Nucl. Phys. A 270 (1976) 489.

[13] S. M. Austin, G. F. Trentelman, and E. Kashy, Astrophys. J. 163 (1971) L79.

[14] H. Stocker, A. A. Rollefson, and C. P. Browne, Phys. Rev. C 4 (1971) 1028.

[15] S. J. McCaslin, F. M. Mann, and R. W. Kavanagh, Phys. Rev. C 7 (1973) 489.

[16] C. A. Barnes, and D. B. Nichols, Nucl. Phys. A 217 (1973) 125.

[17] P. L. Jolivette, J. D. Goss, A. A. Rollefson, and C. P. Browne, Phys. Rev. C 10 (1974) 2629.

[18] J. A. Nolen, and S. M. Austin, Phys. Rev. C 13 (1976) 1773.

[19] D. E. Alburger, Phys. Rev. 118 (1960) 235.

[20] R. G. H. Robertson, R. A. Warner, and S. M. Austin, Phys. Rev. C 15 (1977) 1072.

[21] D. E. Alburger, Phys. Rev. C 16 (1977) 2394.

[22] P. Strehl, Z. Phys. 234 (1970) 416.

[23] H. Crannell, T. A. Griffy, L. R. Suelzle, and M. R. Yearian, Nucl. Phys. A 90 (1967) 152.

[24] H. Crannell et al., Nucl. Phys. A 758 (2005) 399c .

[25] S. E. Woosley, A. Heger, T. Rauscher, and R. D. Hoffman, Nucl. Phys. A 718 (2003) 3c. 